\title{
Multi-Scenario Evaluation of the Direct Method in Strapdown Airborne and Shipborne Gravimetry
}

\author{
Felix Johann, David Becker, Matthias Becker, and E. Sinem Ince
}

\begin{abstract}
In recent years, it was shown that the quality of strapdown airborne gravimetry using a navigation-grade strapdown inertial measurement unit (IMU) could be on par with "classical" airborne gravimeters as the 2-axis stabilized LaCoste and Romberg S-type gravimeter. Basically, two processing approaches exist in strapdown gravimetry. Applying the indirect method (also referred to as "inertial navigation approach" or "one-step approach"), all observations - raw GNSS observations or position solutions, IMU specific force and angular rate measurements - are combined in a single Kalman Filter. Alternatively, applying the direct method (also referred to as "accelerometry approach" or "cascaded approach"), GNSS position solutions are numerically differentiated twice to get the vehicle's kinematic acceleration, which is then directly removed from the IMU specific force measurement in order to obtain gravity. In the scope of this paper, test runs for the application of strapdown airborne and shipborne gravimetry are evaluated using an iMAR iNAV-RQH-1003 IMU. Results of the direct and the indirect methods are compared to each other. Additionally, a short introduction to the processing scheme of the Chekan-AM gravimeter data is given and differences between Chekan-AM and strapdown results of the shipborne campaigns are analysed. Using the same data set, the cross-over residuals suggest a similar accuracy of $0.39 \mathrm{mGal}$ for the Chekan-AM and $0.41 \mathrm{mGal}$ for the adjusted strapdown results (direct method).
\end{abstract}

Keywords

Airborne gravimetry $\cdot$ Gravity disturbance $\cdot$ IMU $\cdot$ Shipborne gravimetry $\cdot$ Strapdown

F. Johann $(\bowtie) \cdot$ M. Becker

Physical and Satellite Geodesy, Technische Universität Darmstadt, Darmstadt, Germany

e-mail: johann@psg.tu-darmstadt.de; becker@psg.tu-darmstadt.de

D. Becker

School of Earth and Planetary Sciences, Curtin University, Perth, WA, Australia

e-mail: david.becker@curtin.edu.au

E. S. Ince

Global Geomonitoring and Gravity Field, Helmholtz-Centre Potsdam, GFZ German Research Center for Geosciences, Potsdam, Germany

e-mail: elmas.sinem.ince@gfz-potsdam.de

\section{Introduction}

Airborne and shipborne gravimetry enables gravity determination with a medium accuracy, compared to satellite and terrestrial gravimetry. Especially in remote areas, where terrestrial measurements are cost-intensive and time-consuming or even impossible, it is widely used to enhance the accuracy and resolution of satellite-based gravity data. In Polar Regions without satellite gravity data, it is the only practicable gravity determination approach.

While shipborne gravimetry using gas-pressured, pendulum or spring gravimeters had already been employed in the first half of the twentieth century (Marson 2012; Nabighian 
et al. 2005), first airborne gravity test flight results using a horizontally stabilized spring gravimeter have not been published before 1960 (Nettleton et al. 1960). In the 1990s, a major improvement in the obtained accuracy followed the advent of the Global Positioning System (GPS), followed by further Global Navigation Satellite Systems (GNSS). The application of inertial measurement units (IMUs) as gravimeters could be shown to be capable of generating results on an accuracy level similar to that of the "classical" approach, where horizontally stabilized spring gravimeters are used (Glennie et al. 2000; Becker et al. 2016).

Main advantages of the IMU usage in the so-called "strapdown" approach are a lower instrument weight, less space and power requirements as well as lowered purchase and maintenance costs. Furthermore, the measurement principle enables vector (3-D) gravimetry and the system is less impaired by turbulences and flight manoeuvres. Strong sensor drifts affecting strapdown gravimetry can be reduced using calibration methods that can lower their impact significantly. It was demonstrated that the application of a simple warm-up calibration of the IMU's vertical accelerometer can eliminate most of the sensor drift (Becker et al. 2015b; Becker 2016).

Even though the vehicle dynamics differ in airborne and shipborne gravimetry, processing strategies virtually agree.

This paper gives an overview on strapdown gravimetry and recent campaigns with participation of the chair of Physical and Satellite Geodesy (PSGD) of the Technical University of Darmstadt. An introduction in the processing approaches in strapdown gravimetry is given in Sect. 2. To enable an evaluation of the presented strapdown result's exterior accuracy, the data processing in "classical" kinematic gravimetry is briefly presented in Sect. 3 using the example of the Chekan-AM gravimeter of the German Research Centre for Geosciences (GFZ Potsdam). The airborne and shipborne campaigns evaluated in the scope of this paper are discussed in Sects. 4 (campaign introduction), 5 (strapdown processing results) and 6 (comparison to Chekan-AM results), followed by the concluding Sect. 7 .

\section{Processing Approaches in Strapdown Gravimetry}

There exist two fundamentally different processing approaches in (kinematic) strapdown gravimetry: the indirect and the direct method. This paper shortly introduces some basics of both approaches; for a more detailed view, the reader is referred to Becker (2016) and Johann et al. (2019).

The "indirect" method as it was introduced by Jekeli (2001) has also been called "inertial navigation approach" (Ayres-Sampaio et al. 2015), "traditional way" (Kwon and
Jekeli 2001) or "one-step approach" (Becker 2016). Here, all observations from an IMU and a GNSS receiver are integrated in a Kalman filter with a state vector including position, velocity, attitude, sensor biases and gravity. In the prediction step, the previous epoch's state vector is propagated using the IMU observations (accelerations and angular velocities). If GNSS observations are available as pseudoranges and carrier phases (tightly coupled integration) or position and velocity solutions (loosely coupled integration), the Kalman filter will form a weighted average between the predicted and the actual observations. The weighting depends on the accuracy ratio of the predicted states and GNSS observations as reflected by the stochastic model.

"Accelerometry approach" (Ayres-Sampaio et al. 2015; Kwon and Jekeli 2001), or "cascaded approach" (Becker 2016) have been alternative terms for the "direct" method. In contrast to the indirect method, where gravity determination is done in the position domain, in the direct method, vertical gravity is directly obtained in the acceleration domain. The gravity disturbance

$$
\boldsymbol{\delta} \boldsymbol{g}^{n}=\ddot{\boldsymbol{r}^{n}}-\boldsymbol{C}_{b}^{n} \boldsymbol{f}^{b}+\boldsymbol{\delta} \boldsymbol{g}_{\mathrm{eot}}^{n}-\boldsymbol{\gamma}^{n}
$$

in the navigation frame being the difference of gravity and normal gravity $\boldsymbol{\gamma}^{n}$ is directly obtained by subtracting the specific force $\boldsymbol{C}_{b}^{n} \boldsymbol{f}^{b}$ from the kinematic acceleration $\ddot{\boldsymbol{r}}^{n}$ (Wei and Schwarz 1998). All these terms are given in the navigation frame $n$ whose origin is defined to be at the IMU's centre of observations with its orthogonal axes pointing towards North, East and Down. The specific force $\boldsymbol{f}^{b}$ as measured by the IMU accelerometers in the body frame $b$ is transformed to the navigation frame by the multiplication with the rotation matrix $\boldsymbol{C}_{b}^{n}$. Alternatively, quaternions can be used to represent the attitude. The GNSS position solutions are numerically differentiated twice in order to obtain the kinematic acceleration. The vehicle's attitude used as input for the rotation matrix is computed in a GNSS/IMU integration algorithm. The addition of the velocity-dependent Eötvös correction $\boldsymbol{\delta} \boldsymbol{g}_{\text {eot }}^{n}$ eliminates the impacts of the Coriolis acceleration and the centrifugal acceleration caused by the movement of the vehicle relative to the Earth. The obtained gravity disturbance needs to be low-pass filtered in order to eliminate high-frequency noise.

On the one hand, the indirect method implements a wellknown optimal estimation procedure, the Kalman filter, and is commonly deemed to be the more rigorous approach (Becker 2016). On the other hand, if the direct method is applied, the GNSS/IMU integration can be performed using available commercial navigation software (e.g. the NovAtel Waypoint InertialExplorer 8.60 used in the scope of this paper), significantly reducing the burden of software development. 
In order to get the best out of the strapdown data, several corrections need to be applied. A detailed description can be found in Johann et al. (2019).

- A bias and a linear drift are removed from the gravity disturbance results by anchoring the results to known reference values at the airports/harbours combined with a linear interpolation between these static periods. Usually, the reference values are obtained from local terrestrial gravity measurements of superior accuracy (about $0.05 \mathrm{mGal}=$ $0.05 \cdot 10^{-5} \mathrm{~m} / \mathrm{s}^{2}$ or better).

- The distance between the IMU's centre of observation and the GNSS antenna phase centre, the so-called "lever arm", needs to be taken into account.

- If no thermal stabilization housing is used, the gravity results of the iMAR iNAV-RQH-1003 IMU can be significantly improved with a thermal calibration. For the campaign analysis at hand, a warm-up calibration of the vertical accelerometer (Becker et al. 2015a) is applied.

- In various strapdown campaigns, the authors have observed an heading-dependent shift of the gravity disturbance estimates. The effect is strongest when heading northwards or southwards (with opposite sign) and weakest when heading westwards or eastwards. In order to attenuate this systematic error, an empiric correction is applied: The cosine of the heading multiplied with a constant is added to the preliminary gravity disturbance results. The constant is selected empirically for the whole gravimetry campaign. The cause for this effect is unknown and is an object of research. Possible explanations might be an uncorrected instrumental error of the iMAR iNAV-RQH-1003 (Becker 2016) or a modelling/approximation error dependent on the current direction of travel.

- Even though gravity disturbance is determinable during turns in strapdown gravimetry, the quality of the results in these track segments is lowered significantly. Therefore, to get accuracy indicators for the best available results and to be less dependent on the campaign trajectory design, only the data of approximately straight track segments ("lines") is included in the quality analysis.

In the scope of this paper, the precision of the results is examined using a cross-over analysis. For this, the survey plan includes intersecting lines. When the intersections are at nearly the same height and one assumes that there is no significant temporal gravity variation, the root mean square (RMS) of the cross-over residuals at these intersections is a measure for the precision of the results. Assuming an equal accuracy on both lines, the RMS error (RMSE), which is given by the RMS divided by $\sqrt{2}$, indicates the uncertainty of the obtained gravity disturbance values at the lines (Forsberg and Olesen 2010; Becker 2016).

Since the gravity disturbance results have already been fixed to the reference values at the airports/harbours, the linear trend is easily removed from the results. If a sufficient number of cross-over points is available, the impact of further drifts during a measurement flight/cruise can be reduced by a cross-over adjustment (also called "levelling"). During the least-squares adjustment, a bias per line is estimated. If the number of cross-over points is low, the resulting precision value is susceptible to being too optimistic, which can be avoided by the use of correction factors (Becker 2016; Johann et al. 2019).

\section{Chekan-AM Data Processing at GFZ}

To enable a comparison with another measurement system, processing methods and some results using a Chekan-AM gravimeter are presented in this paper in addition to the strapdown analysis.

Since 2011, the GFZ Potsdam has been involved in various shipborne and airborne gravimetry campaigns with its mobile gravimeter Chekan-AM whose working principle is based on angle variation measurements of two quartz sensors that are positioned in a viscous liquid. The performance of this equipment has been verified in GFZ's marine test campaign in Lake Müritz, and other dedicated campaigns such as Lake Constance and the GEOHALO mission (Petrovic et al. 2015). The same equipment is further reliably used in the shipborne gravimetry campaigns in the Baltic Sea within the subactivity 2.1 of the "Finalising Surveys for the Baltic Motorways of the Sea" (FAMOS) project (Swedish Maritime Organisation 2019).

The recovery of the measurements in terms of accelerations is based on the mathematical model and calibration constants provided by the manufacturer Electropribor, St. Petersburg. It is known that the survey vehicle and the measurement conditions have a strong impact on the gravimeter measurements. In principle, the processing scheme presented in Krasnov et al. (2011) is followed in GFZ's data processing routine but the processing needs to be adopted based on the quality of the raw measurements and measurement conditions. Obviously, in mobile gravimeter measurements, the raw recordings need to be converted into acceleration units. Like in strapdown gravimetry, measurements need to be corrected for the Eötvös effect and, most importantly, low-pass filtered to eliminate the high frequency noise components and retrieve meaningful results. Occasionally, different low pass filter lengths can be applied to different quality of datasets. Based on their experiences, GFZ found that, in shipborne gravimetry, a filter length of $400 \mathrm{~s}$ provides satisfying results in terms of measurement accuracy w.r.t. global models and other campaign results computed in a cross-over analysis and is a good tradeoff between the spatial resolution and final data accuracy (Lu et al. 2019). 
One of the most challenging tasks of relative gravimetry is the drift estimation of the gravimeter, which needs to be computed as good as possible and taken into account in the data processing. The gravimeter recordings at the harbours, where reference gravity values are available, are taken before and after completing relevant tracks and are used to compute the drift behaviour of the instrument sensors. After correcting the drift, since the measured values are not absolute gravity but gravity differences measured along the measurement track they need to be tied to absolute gravity values at reference stations that are ideally co-located to the harbour tie points.

It is worth mentioning that the Chekan-AM measurements even after applying a low pass filter are not of very high quality during the turns of the ship. Therefore, these periods lasting about from few to tens of minutes as well as disturbed measurements due to harsh sea conditions or instrumental errors (e.g. unexpected drift behaviour, sensor aging) are manually detected and removed from the final delivered products. Good quality measurements (e.g. performed under optimum measurement conditions during dedicated gravimetry campaigns) may not require detailed investigations.

\section{$4 \quad$ Investigated Airborne and Shipborne Strapdown Gravimetry Campaigns}

Since 2013, PSGD co-organized or supported various strapdown gravimetry campaigns. An IMU of the type iMAR iNAV-RQH-1003 (Fig. 1, bottom right) (IMAR Navigation 2012) was used as gravimeter. The IMU was connected to GNSS antennas for the purpose of time synchronization. The following campaign results are presented in this paper:

- "MY2014": In August 2014, 12 flights were undertaken above the South China Sea northwest of Borneo, Malaysia, using a medium-size aircraft, type Beechcraft King Air 350 (Fig. 2a), with autopilot. For details,

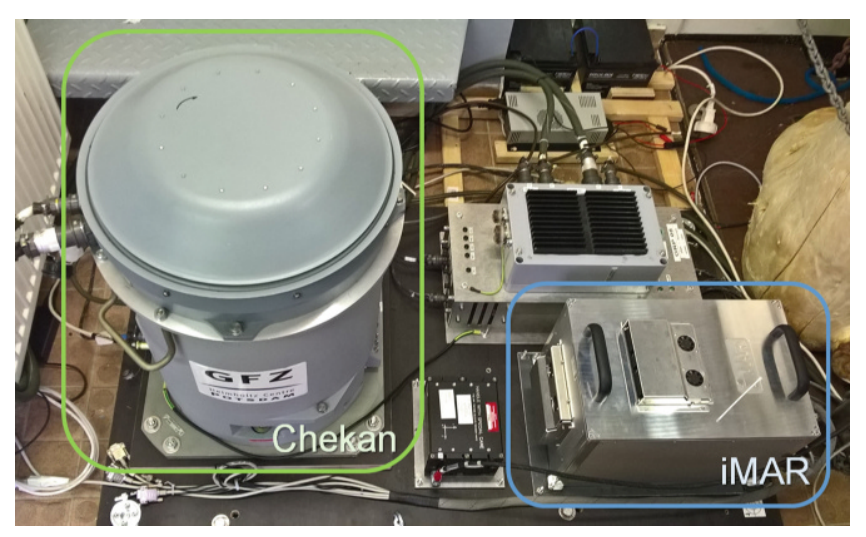

Fig. 1 Chekan-AM and iMAR iNAV-RQH-1003 with iTempStabAddOn aboard DENEB 2018 including a scatter plot of the results and an elaborate evaluation, see (Johann et al. 2019).

- “ODW2017”: In May 2017, a small-scale survey was arranged with a motor glider of the type Grob G 109B (Fig. 2b). The flights were conducted at the Upper Rhine Graben above the Odenwald, a low mountain range with significant gravity variations, located in the southeast of Darmstadt, Germany. An objective of the flight was to test the performance of strapdown gravimetry under difficult conditions with a relatively small aircraft flying in drape mode (following the topography's altitude changes) without autopilot. Due to the small weight of the aircraft and the missing autopilot, the flight has been much less stable than MY2014. GNSS data acquisition was affected by vibrations, possibly also deformations, of the wing, where the antenna was placed on.

- "ODW2018": In March 2018, a test flight was conducted above the same region with a Cessna 206 "Stationair 6" (Fig. 2c), a light aircraft of medium size compared to the aircrafts of MY2014 and ODW2017. Compared to ODW2017, the aircraft change allowed for a smoother flight, but sensor drifts caused by temperature changes had a greater impact in ODW2018 since temperatures before the start were very low, being near the freezing point.

- "BTS2017": Among other campaigns, GFZ performed a dedicated survey in July 2017 on the German Federal Maritime and Hydrographic Agency's (BSH) survey, wreck-search and research vessel DENEB (Fig. 2d). The DENEB campaign measurement tracks were planned by the Federal Agency for Cartography and Geodesy (BKG) considering the gravity measurement densification needs for the determination of a high accuracy German quasigeoid and the gravity measurements were collected by the GFZ's Chekan-AM relative gravimeter. Additionally, in order to investigate the potential of strapdown shipborne gravimetry using navigation-grade IMUs, PSGD's iMAR iNAV-RQH-1003 was installed aboard the DENEB sideby-side with the Chekan-AM. The geographical focus of the 2017 campaign was the Bay of Mecklenburg, and an area between the German island Rügen and the Danish islands Bornholm and Møn. Compared to airborne gravimetry, the Baltic Sea campaigns are challenging for strapdown gravimetry since some cruises (runs from harbour to harbour) are extraordinarily long (up to $60 \mathrm{~h}$ ).

- "BTS2018": In July/August 2018, the mission of the DENEB was continued with another campaign that focussed on the western (Bay of Kiel) and eastern areas (Bay of Pomerania including Polish territory) of the German part of the Baltic Sea. In comparison to the 2017 campaign, the strapdown measurement system was upgraded by encasing the iMAR iNAV-RQH-1003 in a 


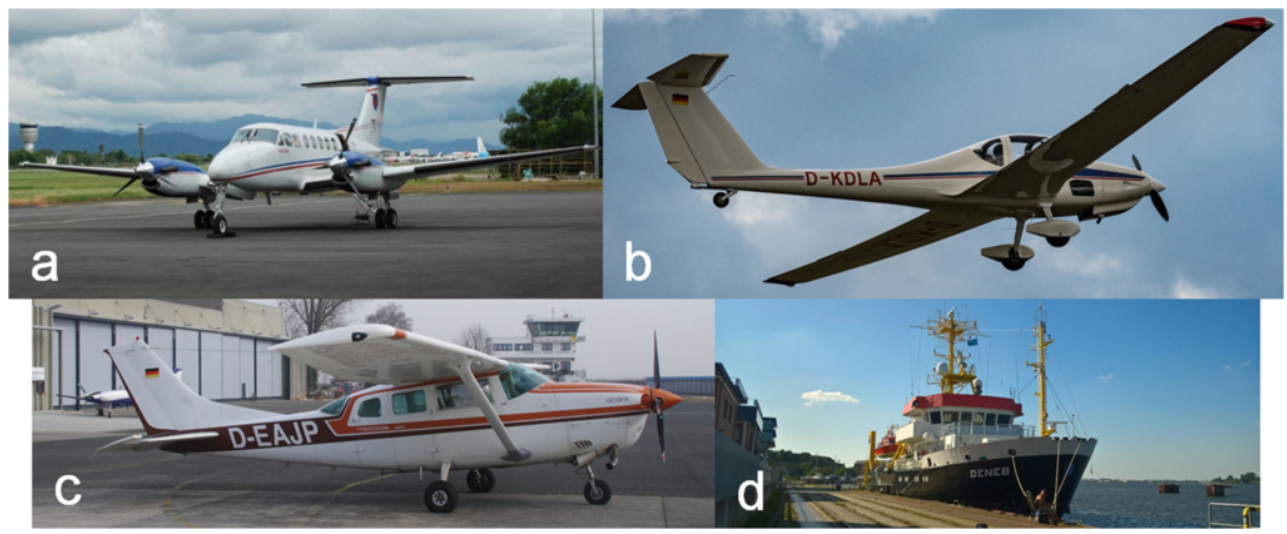

Fig. 2 Vehicles: (a) Beechcraft King Air 350 (MY2014); (b) Grob G109B (ODW2017); (c) Cessna 206 "Stationair 6" (ODW2018); (d) German research vessel DENEB (BTS2017/18)

Table 1 Strapdown gravimetry campaign overview (MY2014 results taken from Johann et al. (2019))

\begin{tabular}{|c|c|c|c|c|c|c|}
\hline & MY2014 & ODW2017 & ODW2018 & \multicolumn{2}{|l|}{ BTS2017 } & BTS2018 \\
\hline Aircraft/vessel & Beechcraft King Air 350 & Grob G109B & Cessna 206 "Stationair 6" & \multicolumn{3}{|l|}{ DENEB } \\
\hline Number of flights/cruises & 12 & 8 & 1 & 4 & $3^{a}$ & 4 \\
\hline Total line distance $[\mathrm{km}]$ & 9,916 & 1,366 & 1,034 & 1,487 & 1,021 & 2,534 \\
\hline Mean line velocity $[\mathrm{m} / \mathrm{s}]$ & 87 & 36 & 54 & 4.7 & 4.6 & 4.8 \\
\hline $\begin{array}{l}\text { Ellipsoidal flight height } \\
(\operatorname{mean} / \min / \max )[\mathrm{m}]\end{array}$ & $1953 / 1016 / 4256$ & $655 / 396 / 927$ & $947 / 797 / 980$ & - & - & - \\
\hline Number of cross-over points & 105 & 429 & 222 & 32 & 20 & 70 \\
\hline Turbulence (RMS-g) $\left[\mathrm{mm} / \mathrm{s}^{2}\right]$ & 235 & 769 & 128 & 65 & 42 & 48 \\
\hline GNSS sampling frequency $[\mathrm{Hz}]$ & 5 & $1 \ldots 20$ & 1 & 1 & 1 & 1 \\
\hline Low-pass filter length (-6dB) [s] & 130 & 130 & 120 & 400 & 400 & 400 \\
\hline Half-wavelength resolution [km] & 5.7 & 2.3 & 3.3 & 0.9 & 0.9 & 1.0 \\
\hline $\begin{array}{l}\text { RMSE non-adjusted [mGal] direct } \\
\text { (indirect) }\end{array}$ & $1.26(1.3)$ & $3.10(3.0)$ & 3.97 & $1.28(1.18)^{\mathrm{b}}$ & 0.81 & 0.71 \\
\hline $\begin{array}{l}\text { RMSE adjusted [mGal] direct } \\
\text { (indirect) }\end{array}$ & $0.62(0.68)$ & $1.12(0.90)$ & 0.64 & $1.00(0.81)^{b, c}$ & $0.86^{\mathrm{c}}$ & $0.55^{\mathrm{c}}$ \\
\hline
\end{tabular}

${ }^{a}$ BTS2017: Three cruises are remaining if one of the four cruises was rejected assuming an outlier

${ }^{\mathrm{b}}$ BTS2017: The heading-dependent correction has not been applied in the indirect method

${ }^{\mathrm{c}}$ The cross-over adjustment of the BTS campaigns was performed stint-wise (complete run from harbour to harbour), while the other campaigns were adjusted line-wise. Usually, lower RMSE values are obtained via line-wise adjustment

housing, the iMAR iTempStab-AddOn, which stabilises the IMU's temperature using two Peltier elements.

Dual frequency GNSS receivers tracking GPS and GLONASS were installed in all campaigns. Positions were calculated in the Precise Point Positioning (PPP) mode with the NovAtel Waypoint InertialExplorer 8.60 using precise satellite orbit and clock products by the Center for Orbit Determination in Europe (CODE). The campaign statistics are summarised in Table 1. The turbulence metric and the obtained precision values (RMSE) will be discussed in Sect. 5 .

\section{$5 \quad$ Strapdown Results}

The obtained vertical gravity disturbance without crossover adjustment for both Odenwald and both Baltic Sea campaigns are illustrated in Figs. 3, 4, 5, 6. For the Malaysia

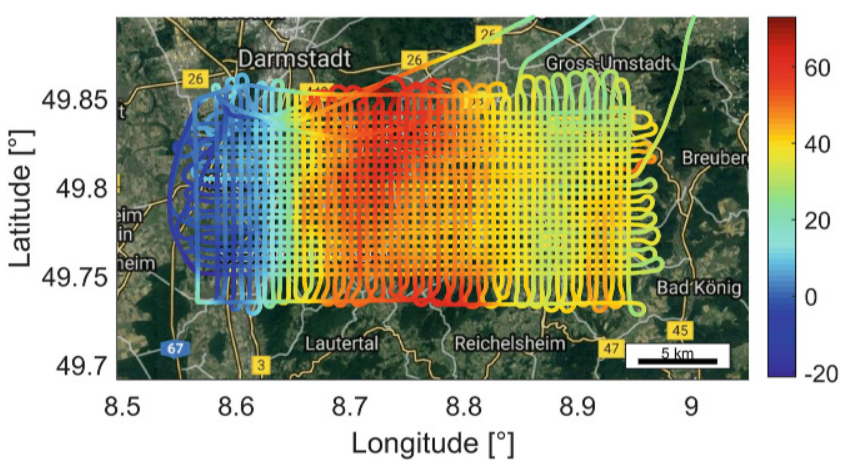

Fig. 3 Vertical gravity disturbance [mGal] ODW2017 (Map data: Google, GeoBasis-DE/BKG, Europa Technologies)

campaign, equivalent figures including preliminary results of the horizontal components can be found in (Johann et al. 2019). RMSE obtained in cross-over analyses with and 


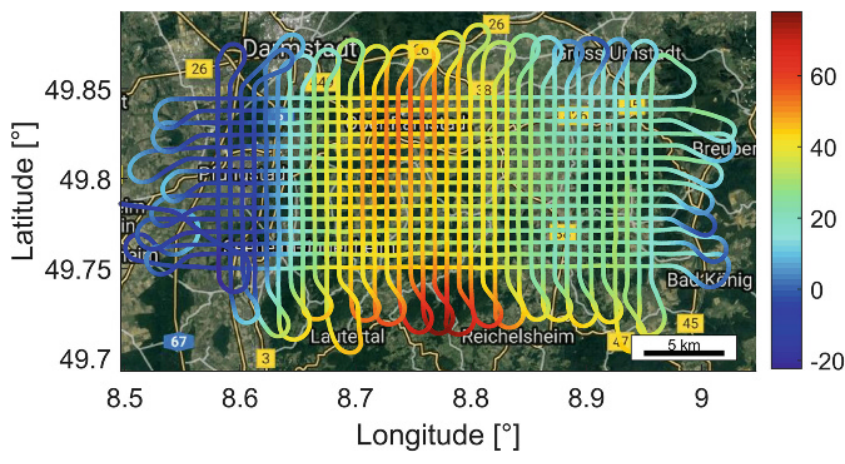

Fig. 4 Vertical gravity disturbance [mGal] ODW2018 (Map data: Google, GeoBasis-DE/BKG, Europa Technologies)

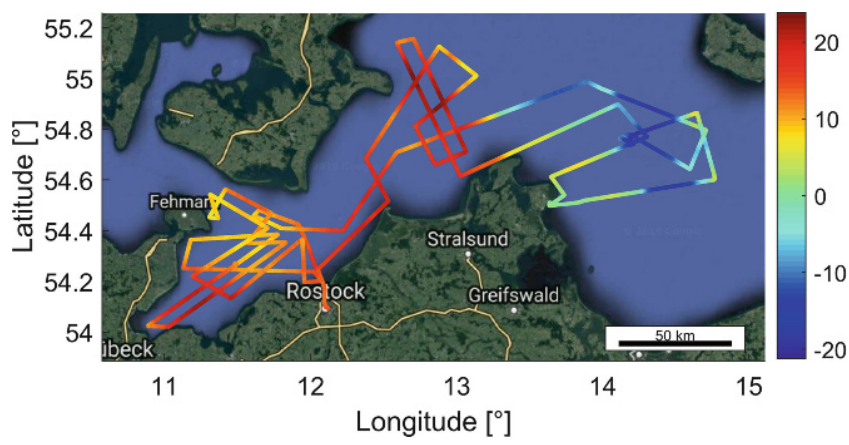

Fig. 5 Vertical gravity disturbance [mGal] BTS2017 (Map data: Google, GeoBasis-DE/BKG, Landsat/Copernicus)

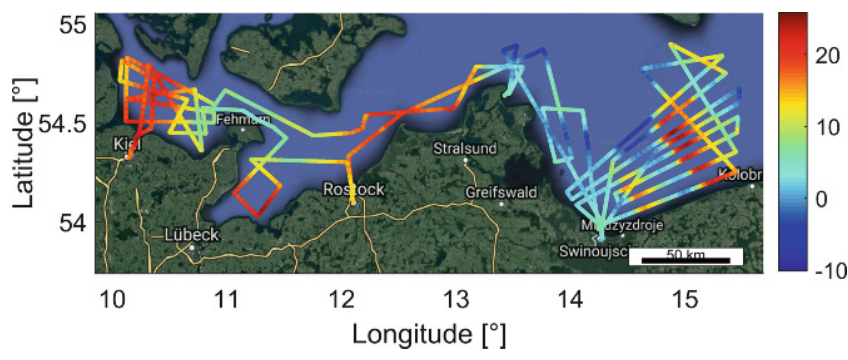

Fig. 6 Vertical gravity disturbance [mGal] BTS2018 (Map data: Google, GeoBasis-DE/BKG, Landsat/Copernicus)

without cross-over adjustment for all four campaigns are noted in Table 1, where available results of the indirect method of strapdown gravimetry are given in parentheses. For the Malaysia 2014 campaign, the results of the direct and the indirect method are on a par with an RMSE of about $1.3 \mathrm{mGal}$ without and $0.65 \mathrm{mGal}$ with line-wise crossover adjustment. For the Odenwald 2017 campaign (Fig. 3), results are considerably worse with an RMSE of $3.1 \mathrm{mGal}$ without and $1.1 \mathrm{mGal}$ with adjustment. The indirect method performs slightly better (3.0 and $0.9 \mathrm{mGal})$. Without adjustment, the results for Odenwald 2018 (Fig. 4) are even worse ( $4.0 \mathrm{mGal})$, maybe caused by temperature calibration problems due to the low environment temperature. In contrast, after adjustment, the RMSE is at the precision level of the Malaysia campaign $(0.64 \mathrm{mGal})$.

For the Baltic Sea 2017 campaign (Fig. 5), without adjustment, an RMSE of $1.28 \mathrm{mGal}$ is obtained applying the direct method and $1.18 \mathrm{mGal}$ applying the indirect method. It should be noted that the empirical heading-dependent correction was not used in the indirect method. Hence, further improvements might be possible. After a cruisewise cross-over adjustment, RMSE improved to $1.00 \mathrm{mGal}$ (direct method) and $0.81 \mathrm{mGal}$ (indirect method). A linewise adjustment was not possible, since isolated nets would occur causing a datum defect. One of the four cruises has been identified as an outlier. The rejection resulted in an improved accuracy of $0.81 \mathrm{mGal}$ without adjustment (direct method). A cruise-wise adjustment did not enhance the results after the outlier rejection. In the Baltic Sea 2018 campaign (Fig. 6) with the installed temperature stabilisation housing, significantly better results with an RMSE of 0.71 $\mathrm{mGal}$ before and $0.55 \mathrm{mGal}$ after the cruise-wise adjustment have been noted. Again, a line-wise adjustment was not applicable. To test the repeatability of the results, both Baltic Sea campaigns have also been examined together (without the outlier cruise in 2017) leading to RMSE of $0.87 \mathrm{mGal}$ without and $0.66 \mathrm{mGal}$ after adjustment, medium accuracies compared to the campaign's single results.

The accuracy of airborne gravimetry results is suspected to depend on the flight turbulence level. A turbulence metric that directly indicates the reaction of the aircraft turbulences is the RMS-g. It is defined as the moving standard deviation of the vertical acceleration during the flight (Becker 2016). Other turbulence metrics like the Eddy Dissipation rate, which rate the atmospheric turbulence state, eliminate some aircraft-dependent effects acting on the IMU. In the scope of this paper, the RMS-g is computed applying a $50 \mathrm{~s}$ moving window on $1 \mathrm{~Hz}$ GNSS vertical acceleration data. For the presented campaigns, a trend between cross-over RMSE and RMS-g might be indicated by the results given in Table 1 if the non-adjusted RMSE of ODW2018 was declared as an outlier caused by temperature calibration issues. Note that accuracy differences depend on multiple campaign parameters like the local gravity field, the low-pass filter length and GNSS data quality.

\section{Comparison to Chekan-AM Results}

Using the cross-over analysis algorithm implemented in the direct method of strapdown gravimetry, an RMSE of 0.33 $\mathrm{mGal}$ and $0.39 \mathrm{mGal}$ resulted for the Chekan-AM data of the Baltic Sea campaigns 2017 and 2018, respectively. To enable a comparison, line segments affected by harsh sea conditions have also been removed from the strapdown results resulting 

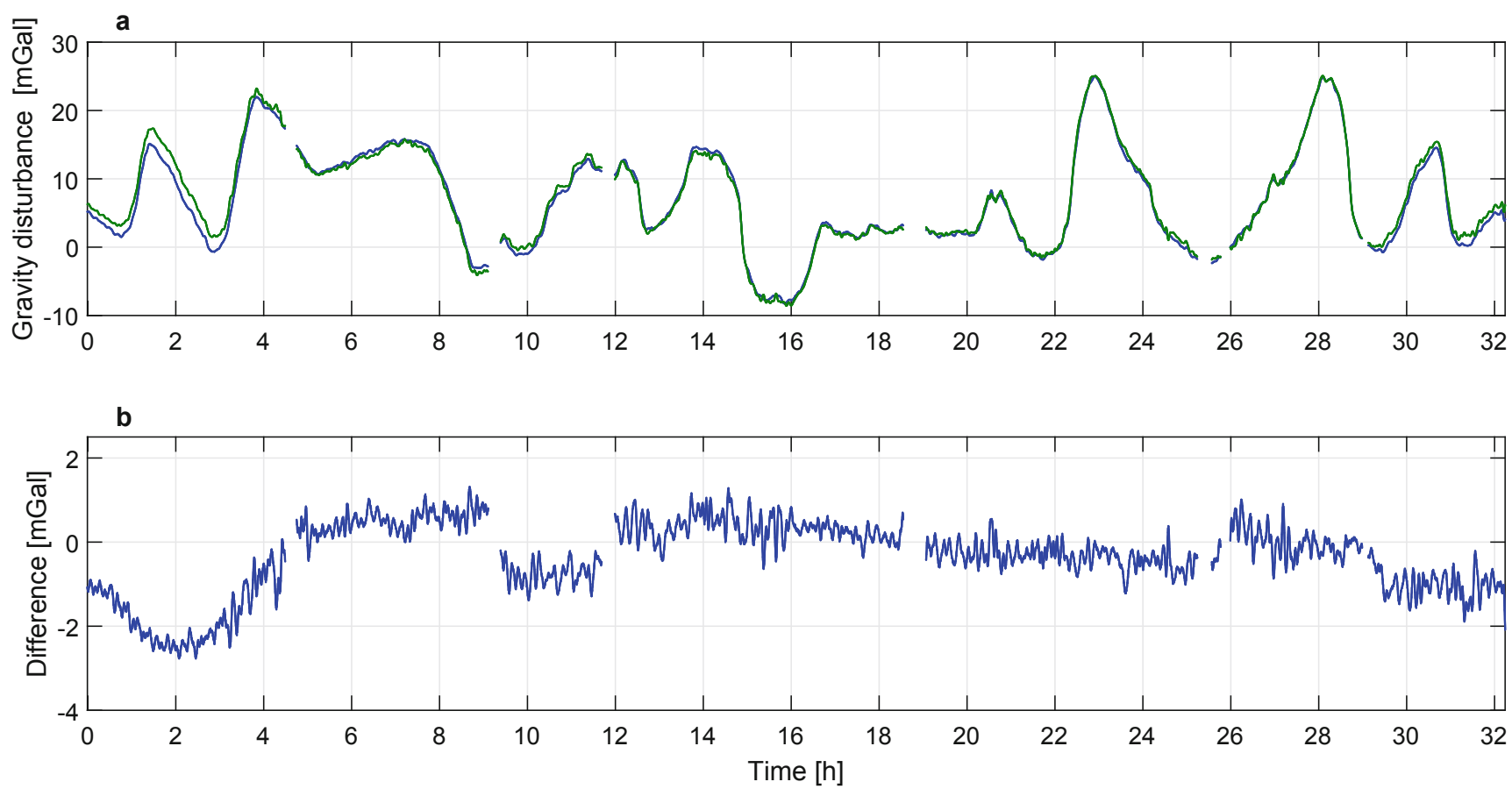

Fig. 7 Comparison of estimated vertical gravity disturbances for BTS2018, cruise 2: (a) blue: strapdown (direct), green: Chekan-AM; (b) Difference (RMS: $0.9 \mathrm{mGal}$ )

in a slightly improved RMSE for BTS2017 (improvement of about $0.1 \mathrm{mGal}$ compared to Table 1). For BTS2018, the strapdown RMSE decreased to $0.52 \mathrm{mGal}$ before and 0.41 mGal after cruise-wise adjustment. Regarding the absolute differences to the non-adjusted strapdown results (direct method), the RMS are $1.24 \mathrm{mGal}$ in 2017 (without the outlier cruise) and $0.97 \mathrm{mGal}$ in 2018. This indicates remaining systematic differences, possibly due to different sensor drift behavior. The comparison of the obtained vertical gravity disturbances of cruise 2, BTS2018, are shown in Fig. 7.

\section{$7 \quad$ Conclusions}

After applying several corrections, the precision of the presented strapdown gravimetry campaigns is between 0.7 and $4.0 \mathrm{mGal}$ without cross-over adjustment. The best RMSE for an airborne campaign $(1.26 \mathrm{mGal})$ was reached at Malaysia 2014, which was conducted with a medium-size aircraft with autopilot under relatively stable flight conditions.

After a line-wise adjustment, the airborne RMSE obtained at Malaysia 2014 and Odenwald 2018 were at the same level (about $0.6 \mathrm{mGal}$ ). The corresponding precision of the Odenwald 2017 campaign is significantly worse $(1.1 \mathrm{mGal})$, probably suffering from GNSS acquisition problems.

The successful combination of both Baltic Sea campaigns indicates a good repeatability of the direct method of strapdown gravimetry. In the implementations created at PSGD, the accuracy of this method is on a par with indirectly obtained results for most evaluated campaigns. Many additional airborne and shipborne surveys processed with both methods would be required to enable statistically firm statements about the accuracy ratio of both approaches under various conditions.

The first test of PSGD's new thermal housing at the Baltic Sea 2018 campaign indicates that its installation improves the long-term stability significantly. Comparing line segments without harsh sea conditions, the precision of the cruise-wise adjusted strapdown gravity disturbance results of $0.41 \mathrm{mGal}$ is competitive with the Chekan-AM results $(0.39 \mathrm{mGal})$. Remaining systematic differences between the strapdown and Chekan results are a subject of research.

Advantages of the strapdown approach like the possibility of vector gravimetry let this approach be an auspicious alternative to the classical approach using horizontally stabilized spring gravimeters.

Acknowledgements The financial support by the Deutsche Forschungsgemeinschaft (DFG) as a part of the project "Innovative calibration methods for strapdown airborne vector gravimetry aboard HALO" within the framework of SPP 1294 "Atmospheric and Earth System Research with the "High Altitude and Long Range Research Aircraft" (HALO)" is gratefully acknowledged. The Malaysia airborne gravity campaign was financed by the Department of Survey and Mapping, Malaysia (JUPEM) and was conducted in cooperation with the Technical University of Denmark (DTU Space). The Odenwald 2017 campaign was conducted by PSGD in cooperation with DTU Space, the Technical University of Dresden (TU Dresden) and the 
Technical University of Munich (TU Munich). The Odenwald 2018 campaign was conducted by iMAR Navigation in cooperation with DTU Space. The DENEB 2017 and 2018 campaigns have been carried out by the German Federal Agency for Cartography and Geodesy (BKG), the German Research Centre for Geosciences (GFZ Potsdam) and the German Federal Maritime and Hydrographic Agency (BSH) within subactivity 2.1 of the project "Finalising Surveys for the Baltic Motorways of the Sea" (FAMOS), co-financed by the European Union. Joachim Schwabe, BKG, adapted the GFZ Chekan-AM results of the 2017 Baltic Sea campaign. The Malaysia airborne gravity campaign was financed by the Department of Survey and Mapping, Malaysia (JUPEM) and was conducted in cooperation with the Technical University of Denmark (DTU Space).

\section{References}

Ayres-Sampaio D, Deurloo R, Bos M, Magalhães A, Bastos L (2015) A comparison between three IMUs for strapdown airborne gravimetry. https://doi.org/10.1007/s10712-015-9323-5

Becker D (2016) Advanced calibration methods for strapdown airborne gravimetry. Phd thesis, Technische Universität Darmstadt, Darmstadt. http://tuprints.ulb.tu-darmstadt.de/5691/

Becker D, Becker M, Leinen S, Zhao Y (2015a) Estimability in strapdown airborne vector gravimetry. In: International association of geodesy symposia. https://doi.org/10.1007/1345_2015_209

Becker D, Nielsen JE, Ayres-Sampaio D, Forsberg R, Becker M, Bastos L (2015b) Drift reduction in strapdown airborne gravimetry using a simple thermal correction. J Geodesy 89(11):1133-1144. https://doi. org/10.1007/s00190-015-0839-8

Becker D, Becker M, Olesen AV, Nielsen JE, Forsberg R (2016) Latest results in strapdown airborne gravimetry using an iMAR RQH unit. In: 4th IAG symposium on terrestrial gravimetry, State Research Center of the Russian Federation, pp 19-25

Forsberg R, Olesen AV (2010) Airborne gravity field determination. In: Xu G (ed) Sciences of geodesy - I: advances and future directions, chap 3, pp 83-104. Springer, Berlin. https://doi.org/10.1007/978-3642-11741-1_3

Glennie CL, Schwarz KP, Bruton AM, Forsberg R, Olesen AV, Keller K (2000) A comparison of stable platform and strapdown airborne gravity. J Geodesy 74(5):383-389. https://doi.org/10.1007/ s001900000082
IMAR Navigation (2012) Inertial measurement system for advanced applications. Tech. rep., St. Ingbert, http://www.imar-navigation.de/ downloads/NAV_RQH_1003_en.pdf

Jekeli C (2001) Inertial navigation systems with geodetic applications. de Gruyter, Berlin

Johann F, Becker D, Becker M, Forsberg R, Kadir M (2019) The direct method in strapdown airborne gravimetry - a review. Zeitschrift für Geodäsie, Geoinformation und Landmanagement 144(5). https://doi. org/10.12902/zfv-0263-2019. https://geodaesie.info/zfv/heftbeitrag/ 8493

Krasnov AA, Sokolov AV, Usov SV (2011) Modern equipment and methods for gravity investigation in hard-to-reach regions. Gyroscopy Navig 2(3):178-183. https://doi.org/10.1134/ s2075108711030072

Kwon JH, Jekeli C (2001) A new approach for airborne vector gravimetry using GPS/INS. J Geodesy 74:690-700. https://doi.org/10.1007/ s001900000130

Lu B, Barthelmes F, Li M, Förste C, Ince ES, Petrovic S, Flechtner F, Schwabe J, Luo Z, Zhong B, He K (2019) Shipborne gravimetry in the Baltic Sea: data processing strategies, crucial findings and preliminary geoid determination tests. J Geodesy. https://doi.org/10. 1007/s00190-018-01225-7

Marson I (2012) A short walk along the gravimeters path. Int J Geophys 2012. https://doi.org/10.1155/2012/687813

Nabighian MN, Ander ME, Grauch VJS, Hansen RO, LaFehr TR, Li Y, Pearson WC, Peirce JW, Phillips JD, Ruder ME (2005) Historical development of the gravity method in exploration. Geophysics 70(6):63ND-89ND. https://doi.org/10.1190/1.2133785. http://library.seg.org/doi/10.1190/1.2133785

Nettleton LL, LaCoste L, Harrison JC (1960) Tests of an airborne gravity meter. Geophysics 25(1). https://doi.org/10.1190/1.1438685

Petrovic S, Barthelmes F, Pflug H (2015) Airborne and shipborne gravimetry at GFZ with emphasis on the GEOHALO project. In: Rizos C, Willis P (eds) International association of geodesy symposia, pp 313-322. Springer, Cham. https://doi.org/10.1007/1345_ 2015_17

Swedish Maritime Organisation (2019) The FAMOS project: For future navigation in the Baltic Sea and beyond. http://www.famosproject. eu/famos/

Wei M, Schwarz KP (1998) Flight test results from a strapdown airborne gravity system. J Geodesy (72):323-332. https://doi.org/10.1007/ s001900050171

Open Access This chapter is licensed under the terms of the Creative Commons Attribution 4.0 International License (http://creativecommons. $\mathrm{org} /$ licenses/by/4.0/), which permits use, sharing, adaptation, distribution and reproduction in any medium or format, as long as you give appropriate credit to the original author(s) and the source, provide a link to the Creative Commons licence and indicate if changes were made.

The images or other third party material in this chapter are included in the chapter's Creative Commons licence, unless indicated otherwise in a credit line to the material. If material is not included in the chapter's Creative Commons licence and your intended use is not permitted by statutory regulation or exceeds the permitted use, you will need to obtain permission directly from the copyright holder.

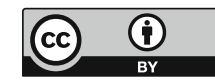

